\title{
Long-Term Vitamin E-Deficient Mice Exhibit Cognitive Dysfunction via Elevation of Brain Oxidation
}

\author{
Koji FuKuI ${ }^{1,2}$, Keisuke NAKAMURA ${ }^{1}$, Masashi SHIRAI ${ }^{1}$, Anna HIRANO $^{1}$, \\ Hirokatsu TAKATSU ${ }^{3}$ and Shiro URANO ${ }^{2}$ \\ ${ }^{1}$ Physiological Chemistry Laboratory, Department of Bioscience and Engineering, College of \\ Systems Engineering and Sciences, Shibaura Institute of Technology, Fukasaku 307, \\ Minuma-ku, Saitama 337-8570, Japan \\ ${ }^{2}$ Life Support Technology Research Center, Research Organization for Advanced Engineering, \\ Shibaura Institute of Technology, Saitama 337-8570, Japan \\ ${ }^{3}$ School of Creative Science and Engineering, Faculty of Science Engineering, Waseda University, \\ 3-4-1 Okubo, Shinjuku-ku, Tokyo 169-8555, Japan
}

(Received March 12, 2015)

\begin{abstract}
Summary Vitamin E inhibits oxidative processes in living tissues. We produced vitamin E-deficient mice by feeding them a vitamin E-deficient diet to verify the influence of chronic vitamin E deficiency on cognitive function. We measured cognitive function over a 5-d period using the Morris water maze task, as well as antioxidant enzyme activity and lipid peroxidation in discrete brain regions, and total serum cholesterol content. Threeand six-mo-old vitamin E-deficient and age-matched control mice were used. In addition, 24-mo-old mice were used as an aged-model. In the 3-mo-old mice, cognitive function in the vitamin E-deficient (short-term vitamin E-deficient) group was significantly impaired compared to age-matched controls. Although the lipid peroxidation products in the cerebral cortex, cerebellum and hippocampus did not significantly differ in 3-mo-old mice, the levels in the 6-mo-old vitamin E-deficient (long-term vitamin E-deficient) mice were significantly increased compared to age-matched controls. Serum cholesterol content was also significantly increased in the short- and long-term vitamin E-deficient mice compared to their respective age-matched controls. These results indicate that chronic vitamin E deficiency may slowly accelerate brain oxidation. Thus, vitamin E concentrations may need to be monitored in order to prevent the risk of cognitive dysfunction, even under normal conditions.
\end{abstract}

Key Words vitamin E-deficient, long-term, cognition, cholesterol, brain oxidation

Reactive oxygen species (ROS) attack living tissues and induce oxidative deterioration (1). It is well known that the elevation of oxidative products in living tissues accelerates the risk of the development and progression of serious diseases such as neurodegenerative disorders $(2,3)$, liver disease $(4,5)$, and heart disease $(6,7)$. These oxidation-related diseases are referred to as free radical diseases. Furthermore, continual consumption of oxygen, a normal physiological process, may be related to the progression of senescence $(1,8,9)$. In the 1950s, Harman proposed a famous hypothesis, the free radical theory of aging (10). In our previous study, lipid peroxidation products such as thiobarbituric acid reactive substances (TBARS) and lipid hydroperoxides (LOOH), increased significantly in aged versus young rodents $(11,12)$. In order to prevent the risk of the development or progression of free radical diseases in the senescence process, it is necessary to attenuate ROS production or oxidative damage. However, the production of ROS in aerobic organisms cannot be eliminated, mainly because it is not possible to prevent the leakage of electrons in the mitochondrial electron transport system

E-mail: koji@sic.shibaura-it.ac.jp
(13). Free electrons rapidly react with oxygen, resulting in the production of superoxide anion radicals in the mitochondria. The antioxidant defense system is capable of attenuating the toxicity of ROS. This system consists mainly of two components. The first component consists of endogenous antioxidant enzymes, such as a superoxide dismutase (SOD), catalase (CAT), and glutathione peroxidase (GPx). The second component consists of food-derived antioxidant substances, such as catechins, polyphenols, and vitamins. Vitamin E ( $\alpha$-tocopherol), which is a natural lipophilic vitamin, possesses a powerful antioxidant function (14-16). Therefore, vitamin E deficiency induces disruption of the balance between production and detoxification of ROS. It is well known that vitamin $\mathrm{E}$ deficiency induces anemia, reduced erythrocyte life span, and ataxia in humans (16-18). Previously, we reported that vitamin E-deficient rats exhibit cognitive dysfunction in the Morris water maze task (19). Furthermore, we found elevated apoptosis in hippocampal neurons (20). The vitamin E deficiency period in that study was $8 \mathrm{wk}$. However, the duration of feeding the vitamin E-deficient diet appeared to be too short to clarify the effect of oxidative damage on living tissues. Certainly, the content of vitamin E in every 
organ in this vitamin E-deficient model decreased significantly compared to that in normal animals $(11,21)$. In humans, overproduction of oxidative stress or deficiency in the antioxidant defense system is typically a chronic condition. In this study, we created long-term vitamin E deficiency in mice and determined cognitive function using the Morris water maze apparatus.

\section{MATERIALS AND METHODS}

Animals and reagents. All experimental animals (C57BL/6, male) were purchased from Japan SLC, Inc. (Hamamatsu, Japan) excepting the 24-mo-old mice. Twenty-four-month-old (C57BL/6, male) mice were obtained from the Tokyo Metropolitan Institute of Gerontology (Tokyo, Japan). In order to verify the influence of chronic vitamin E-deficiency, we produced vitamin E-deficient mice by providing the animals with a vitamin E deficient-diet starting at 1 mo of age. In these experiments, the model of vitamin E-deficiency involved rearing mice on the vitamin E-deficient diet until 3 or 6 mo of age. All mice were maintained under controlled conditions of temperature $\left(22 \pm 2^{\circ} \mathrm{C}\right)$ and a $12 \mathrm{~h}$ light/dark cycle, with free access to food and water. The aged-mice were acclimated to their new environment for $1 \mathrm{wk}$ before starting the cognitive trials. The powdered vitamin E-deficient diet (AIN-76A) and normal diet pellets (Labo MR Stock) were purchased from Funabashi Farm Co., Ltd. (Chiba, Japan) and Nosan Corp. (Kanagawa, Japan), respectively. The vitamin E-deficient diet was prepared for consumption by mixing $100 \mathrm{~g}$ of the powdered diet with $20 \mathrm{~mL}$ of vitamin E-depleted corn oil (Funabashi Farm). Samples of brain regions (cerebral cortex, cerebellum and hippocampus) were prepared for biochemical analysis following cognitive testing, as described previously with some modifications (21).

All other chemical reagents were obtained from either Wako Pure Chemical Industries, Ltd. (Osaka, Japan) or Sigma-Aldrich Corp. (St. Louis, MO). All animal experiments were performed with the approval of the Animal Protection and Ethics Committee of the Shibaura Institute of Technology (Tokyo, Japan).

Cognitive performance. Cognitive function was assessed using a Morris water maze apparatus (19, 22). The maze apparatus $(140 \mathrm{~cm}$ in diameter and $45 \mathrm{~cm}$ in height) consists of a pool of constructed acrylic resin. The bottom of the pool was divided into four quadrants by lines and was set up with four different visible marks positioned around the pool. A submerged platform was placed in the center of one quadrant. The water temperature of the pool was maintained at $22 \pm 2^{\circ} \mathrm{C}$. Before starting the cognitive performance trials, the animals were acclimated to the pool and handling by the experimenter by being allowed to swim freely for $60 \mathrm{~s}$ in the absence of a platform and handled over a 3-d period. The cognitive trials were performed 4 times per day and continued for 5 consecutive days. All trials were performed at the same time of day, and carried out every $3 \mathrm{~h}$ (starting at 9:00, 12:00, 15:00, and 18:00). We performed a total of 20 trials per mouse. The platform was maintained in the same location of the pool for all trials. The escape latency (time to reach the goal), swimming distance, swimming speed, and the proportion of time spent swimming in the quadrant containing the platform were measured using ANY-maze software (Stoelting Co., Wood Dale, IL). The learning ability on each day was assessed by calculating the average escape latency of the four daily trials.

Total serum cholesterol content. Total serum cholesterol levels were measured using a commercial kit (Cholesterol E-test Wako), according to the manufacturer's protocol. The oxidation of cholesterol, by cholesterol oxidase, results in the production of hydrogen peroxide. $\mathrm{N}$-Ethyl-N-(2-hydroxy-3-sulfopropyl)-3,5-dimethoxyaniline, sodium salt, $\mathrm{H}_{2} \mathrm{O}_{2}$, and 4-aminoantipyrine undergo oxidative condensation in the presence of peroxidase, which results in the production of a blue dye that can be measured by monitoring absorbance at $600 \mathrm{~nm}$ using a spectrophotometer.

Measurement of antioxidative enzyme activities. The measurement of superoxide dismutase (SOD) activity was performed using an assay kit (SOD-Test Wako) according to the manufacturer's protocol. This method is based on the xanthine oxidase reaction. The xanthine oxidase reaction induces superoxide. Nitroblue tetrazolium reacts with superoxide and is reduced to diformazan. However, in the presence of SOD, superoxide reacts with SOD and results in the production of hydrogen peroxide. As a result, the production of diformazan is decreased. In this assay, SOD activity is expressed as the ratio of decreased diformazan. SOD activity was determined by measuring absorbance at $560 \mathrm{~nm}$ using a spectrophotometer.

Glutathione peroxidase (GPx) activity can be determined by monitoring $\beta$-nicotinamide adenine dinucleotide phosphate (NADPH) levels. Sample homogenates were mixed with reduced glutathione (GSH), glutathione reductase (GR), and NADPH, which was then rapidly incubated with hydrogen peroxide. Hydrogen peroxide is reduced by GPx, while GSH is oxidized to glutathione (GSSG). GR reduces GSSG to GSH and concomitantly oxidizes NADPH to $\mathrm{NADP}^{+}$. The levels of NADPH were measured by monitoring absorbance at $340 \mathrm{~nm}$.

Analysis of lipid peroxidation. To analyze lipid oxidation, we measured thiobarbituric acid reactive substances (TBARS). Lipid peroxides were measured using Yagi's method, as previously described with some modifications (23). One hundred microliters of the sample homogenates was mixed with $200 \mu \mathrm{L}$ of $5 \mathrm{~mm}$ EDTA, $2 \mathrm{~mL}$ of $1 \%$ phosphate acid, and $1 \mathrm{~mL}$ of $0.7 \%$ thiobarbituric acid. The mixture was heated to $100^{\circ} \mathrm{C}$ in a block heater for $45 \mathrm{~min}$. After cooling on ice, the sample was incubated with $2 \mathrm{~mL}$ of butanol for $3 \mathrm{~min}$. Following centrifugation, the upper layer was isolated and absorbance at $535 \mathrm{~nm}$ was measured using a spectrophotometer (GeneQuant 100, GE Healthcare UK Ltd., Little Chalfont, UK). This oxidized marker was expressed as per mg protein in the samples.

Statistical analysis. Data were plotted as the mean \pm SE and analyzed using Student's t-test, with $p<0.05$ considered significant. Cognitive performance data were 


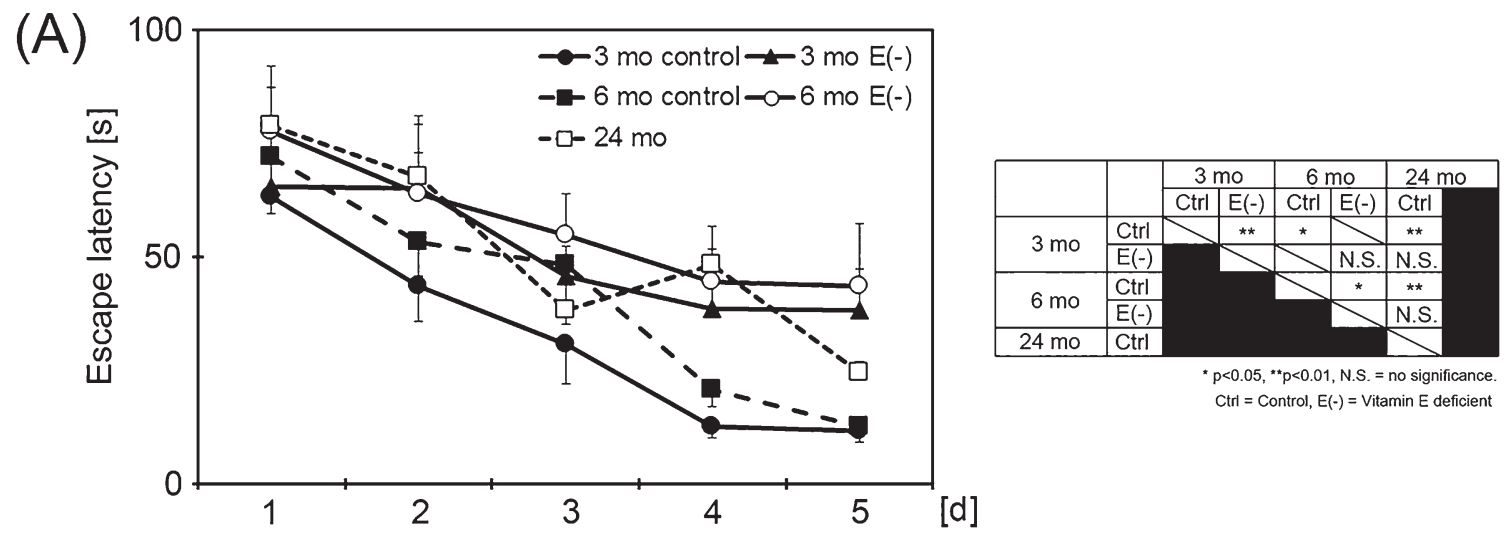

(B)

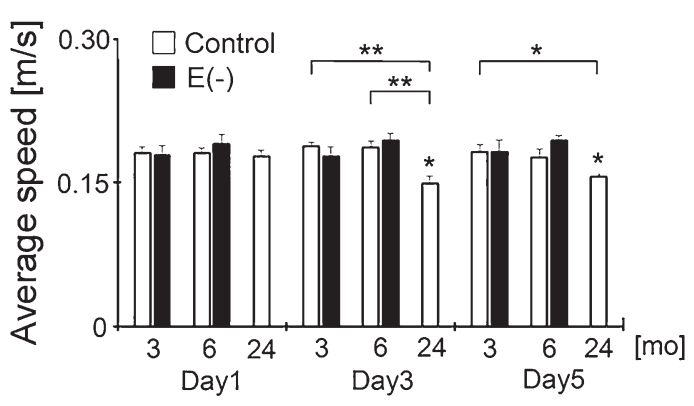

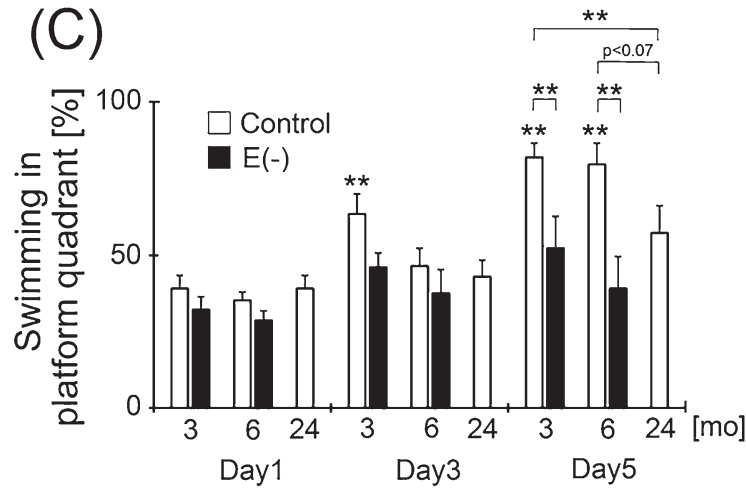

Fig. 1. Short periods of dietary vitamin E-deficiency induce cognitive dysfunction in mice. The time required to reach the goal (escape latency) is shown in panel A. The maximum swimming duration was $60 \mathrm{~s}$. Trials were performed on 5 consecutive days and 4 trials were conducted per day, with daily trials performed every $3 \mathrm{~h}$. Learning ability on a given day was assessed by calculating the average escape latency of the 4 daily trials. The right side of panel A shows the result of the statistical analysis. The average swimming speeds are shown B. The proportions of time spent swimming in the quadrant containing the platform are shown in panel C. Asterisks placed directly above at bar indicate a significant difference from day 1 in the same group. 3-mo control, $n=8$; 3-mo vitamin E-deficient, $n=6$; 6 -mo control, $n=6$; 6 -mo vitamin E-deficient, $n=6 ; 24-\mathrm{mo}, n=4$. The data in panel A was analyzed using two-way ANOVA. The data in panel B and C were analyzed using Student's t-tests; ${ }^{*} p<0.05,{ }^{* *} p<0.01$.

analyzed using a two-way factorial analysis of variance (two-way ANOVA), with $p<0.05$ considered significant.

\section{RESULTS}

Cognitive function is impaired in short- and long-term vitamin E-deficient mice

In the Morris water maze trial, the escape latency (arrival time at the "escape" platform) gradually decreased in all groups (Fig. 1A). This result showed that the mice in each group gradually learned the platform location. Statistical analysis (two-way ANOVA) indicated that the escape latencies of both vitamin E-deficient groups were significantly longer than those of the age-matched controls. Although no significant difference between 3- and 6-mo-old controls was observed, the escape latency of the 3-mo-old mice was less than that of the 6-mo-old mice. The escape latency of agedmice was significantly longer than that of the 3 and 6 mo-old controls. However, there was no significant difference between the escape latencies of the aged-mice and either of the vitamin E-deficient groups. In comparing the vitamin E-deficient models, the escape latency of the 6-mo-old group tended to be longer than that of the 3-mo-old group. The escape latency of the aged-mice on the final day showed a moderate score between the control and vitamin E-deficient mice.

In order to compare the motor function among the five groups, swimming speed in each group was measured. No significant difference was observed in swimming speed between control and vitamin E-deficient mice and age-matched controls (Fig. 1B). On the other hand, the swimming speed of aged-mice significantly decreased on days 3 and 5 compared to the 3-mo-old controls. The ratio of swimming time in the platform area was also similar to the escape latency results (Fig. 1C). On day 5 , the proportion of swimming time in the platform quadrant, was remarkably lower in vitamin E-deficient mice compared to age-matched controls. Although the proportion of swimming time in the platform quadrant in 6-mo-old mice was not significantly different between day 3 , and day 1 , there was a significant difference in 3-mo-old controls. The swimming distances decreased remarkably in the control mice in a time-dependent manner (Fig. 2). In contrast, the swimming distances of vitamin E-deficient mice were remarkably longer than those of the age-matched controls. The average daily 
swimming distance for each group was also similar to the escape latency result (data not shown). These results indicate that vitamin E deficiency tends to induce cognitive dysfunction in mice, according to the Morris water maze task.

3 mo Control
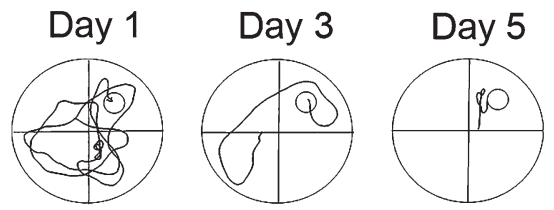

$3 \mathrm{mo} E(-)$
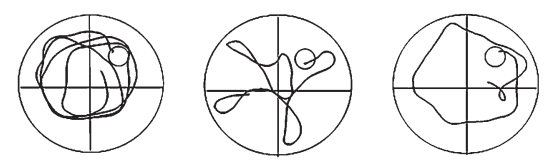

6 mo Control
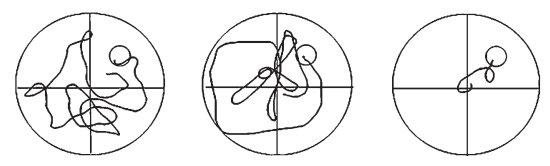

6 mo $E(-)$
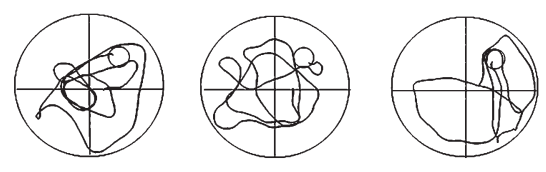

$24 \mathrm{mo}$
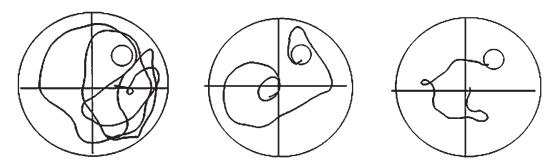

Fig. 2. The Morris water maze swimming trajectories of individual mice from each group. The swimming trajectories of each animal were recorded with a camera placed over the equipment, and were analyzed using ANY-maze software.
Vitamin E deficiency increases serum cholesterol levels

Total serum cholesterol levels were determined in order to verify the mechanism of cognitive dysfunction in long-term vitamin E-deficient mice. As shown in Fig. 3 , total cholesterol levels in vitamin E-deficient mice increased significantly in 3- and 6-mo-old mice compared to their respective age-matched controls. However, total cholesterol levels did not significantly differ between the vitamin E-deficient groups. This result indicates that short periods of vitamin E deficiency induce elevation of serum cholesterol levels. However, a direct correlation was not demonstrated. Further investigation is needed to clarify the relationship between changes in serum cholesterol levels and vitamin E-deficiencyinduced cognitive dysfunction.

Long-term vitamin E-deficient mice tend to exhibit increased lipid peroxidation in the brain

To determine the relationship between long-term vitamin E deficiency-induced cognitive dysfunction and

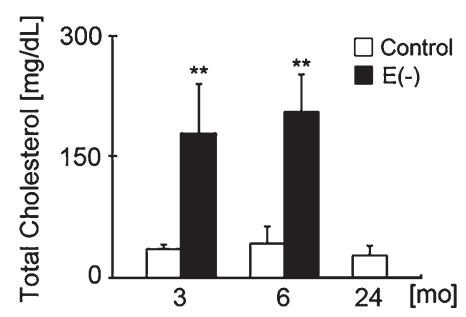

Fig. 3. Vitamin E-deficiency increased serum cholesterol in mice. Asterisks indicate comparison with agematched controls. 3-mo control, $n=8$; 3-mo vitamin E-deficient, $n=6 ; 6$-mo control, $n=6 ; 6$-mo vitamin E-deficient, $n=6 ; 24-\mathrm{mo}, n=4$. The data was analyzed using Student's $t$-tests; ${ }^{*} p<0.05,{ }^{* *} p<0.01$.

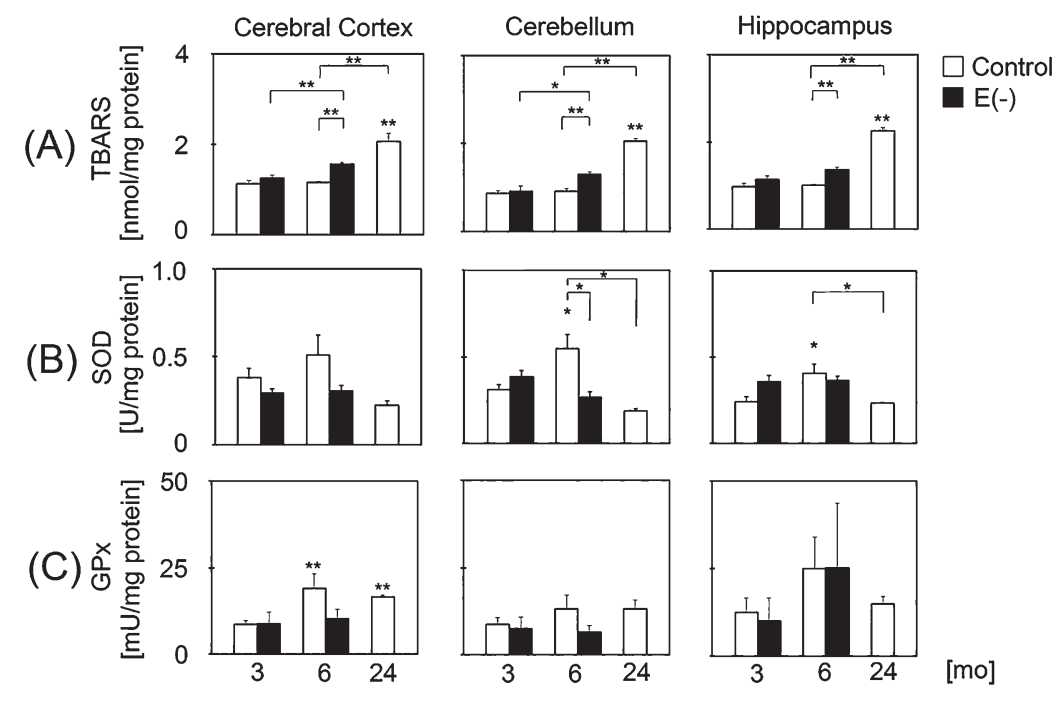

Fig. 4. The effect of vitamin E-deficiency on antioxidant enzyme activity and lipid peroxidation levels in murine brain. Lipid peroxidation was assessed by measuring the levels of thiobarbituric acid reactive substances (TBARS) (A). The activities of the antioxidant enzymes super oxide dismutase (SOD) (B) and glutathione peroxidase (GPx) (C) were measured. All parameters were measured in the cerebral cortex, cerebellum, and hippocampus. Asterisks placed directly above the bars indicate a significant difference from the 3-mo-old control group. 3-mo control, $n=8$; 3-mo vitamin E-deficient, $n=6$; 6-mo control, $n=6$; 6 -mo vitamin E-deficient, $n=6$; 24 -mo, $n=4$. The data was analyzed using Student's $t$-tests. ${ }^{*} p<0.05$, ** $p<0.01$. 
brain oxidation, we measured lipid peroxidation levels and antioxidant enzyme activities. As shown in Fig. 4, TBARS content in aged-mice increased significantly in all brain regions compared to 3-mo-old controls (A). The TBARS content of 6-mo-old vitamin E-deficient mice, but not 3-mo-old vitamin E-deficient mice, increased significantly in all regions compared to age-matched controls. In vitamin E-deficient mice, TBARS content was significantly increased in the cortex and cerebellum of 6-mo-old mice compared to 3-mo-old mice. No significant difference was observed between aged- and vitamin E-deficient mice in any brain region.

With respect to antioxidant enzyme activities, SOD activity in the cerebellum and hippocampus of 6-moold controls was significantly increased compared to that in 3-mo-old controls (B). On the other hand, SOD activity in the cerebellum and hippocampus of agedmice was significantly decreased compared to that in 6-mo-old controls. Contrary to expectations, SOD activity in 6-mo-old mice was only significantly decreased in the cerebellum of vitamin E-deficient animals compared to the same area of age-matched controls. SOD activity was not significantly different between vitamin E-deficient and aged-mice in any brain region. Although GPx activity in vitamin E-deficient mice tended to decrease compared to that in age-matched controls, there was no significant difference between control and vitamin E-deficient mice $(\mathrm{C})$. In the cerebral cortex of control mice, GPx activity in 6-mo-old and aged-mice was significantly increased compared to that in 3-mo-old mice. In the control groups, the activity of antioxidant enzymes in all regions of the 6-mo-old mice tended to be greater than that in the other age groups.

\section{DISCUSSION}

Cognitive impairment occurs with short periods of vitamin E deficiency

It is well known that vitamin $\mathrm{E}$ has a number of beneficial functions in our body. $\alpha$-Tocopherol has been used therapeutically for hepatitis (24), and $\gamma$-tocopherol is used to counteract the inflammatory response (14). Furthermore, $\alpha$-tocopheryl acetate ester has been prescribed to improve peripheral circulatory disorders in Japan. In many cases, including the above, studies of vitamin $\mathrm{E}$ have been based on its antioxidant function. On the other hand, some reports have shown the influences of a vitamin E deficiency. It has been reported that motor functions were impaired in $\alpha$-tocopherol transfer protein knockout mice (25), and induced anxiety behavior was observed in $\alpha$-tocopherol-deficient mice using the elevated plus-maze test (26). These phenomena are based on elevated oxidative stress via a lack or deficiency of vitamin E. Previously, we also reported cognitive dysfunction in vitamin E-deficient rats (19) and detected neuronal apoptosis in the hippocampal CA1 region (20). The results from these studies of vitamin E-deficient rats, including increased apoptosis, were similar to those for aged-rats with normal vitamin E levels. Oxidative stress may be related to the induction of age-related cognitive dysfunction. However, the duration of exposure to the vitamin E-deficient diet was about 2 mo in our previous study. It is possible that only a short period is required for producing vitamin $\mathrm{E}$ deficiency in rodents. However, it was difficult to determine whether the measured results were truly dependent on vitamin E deficiency. In humans, elevated oxidative stress or attenuation of the antioxidant defense system is typically the result of chronic conditions.

In the present study, we investigated the influences of long-term administration of a vitamin E-deficient diet on cognitive function and antioxidant enzyme activities in mice. As expected, cognitive function in the shortterm vitamin E-deficient mice was impaired compared to that in age-matched controls. This result indicates that only a few weeks of vitamin E-deficiency ( $8 \mathrm{wk}$ ) has the potential to induce cognitive dysfunction. On the other hand, the swimming speed of aged-mice was significantly decreased compared to that of 3-and 6-mo-old controls at day 3 and 5. In calculating swimming speed, we have excluded the time spent floating and stopping in the pool. Although motor dysfunction is a potential factor in cognitive dysfunction in aged-mice, we do not think that it is a significant factor. This is because the lipid peroxidation products in all brain regions were significantly increased compared to those in the young controls, and the proportion of time spent swimming in the platform quadrant in aged-mice was not increased during trials. Therefore, the cause of the cognitive dysfunction in aged-mice is likely to be related to brain oxidation.

Long-term vitamin E-deficiency accelerates brain oxidation

Although we could not identify a significant difference, the cognitive function in the long-term vitamin E-deficient mice was remarkably impaired compared to that in short-term deficiency, especially after day 3. The swimming speed of age-matched mice did not differ between the control and vitamin E-deficient groups. This result indicates that learning impairment in vitamin E-deficient mice was not related to motor dysfunction. The chronic vitamin E-deficiency in our experimental model induces gradual and definite cognitive impairment. Previously, we reported the effects of short- and long-term vitamin E-deficiency on $\alpha$-tocopherol content in the cerebral cortex, cerebellum, and hippocampus in mice. Vitamin E levels in the long-term vitamin E-deficient mice were below the detection limit of high performance liquid chromatography (HPLC) with electrochemical detection (21). Furthermore, we assessed the expression of microtubule-associated protein (MAP) light chain (LC) 3 in the cerebral cortex, cerebellum, and hippocampus using Western blotting (21). The expression of MAP LC3-II, which is a major hallmark of autophagy, was significantly decreased in the longterm vitamin E-deficient mice compared to that in shortterm deficiency. On the other hand, the expression of MAP LC3-II in the short-term vitamin E-deficient mice increased significantly compared to that in age-matched controls. Therefore, the mice in the short-term vitamin E deficiency group might be resistant to oxidative stress, due to their young age and short deficiency period. In 
the present study, TBARS content in the cerebral cortex, cerebellum and hippocampus of 3-mo-old mice did not differ significantly between control and vitamin E-deficiency. Conversely, TBARS levels in 6-mo-old mice were significantly different between vitamin E-deficient and control mice.

However, antioxidant enzyme activities in the longterm vitamin E-deficient mice were not significantly different than in age-matched controls, with the exception of SOD activity in the cerebellum. This observation may be due to the SOD and GPx activity measurement kits utilized in the present study, which were not isoform specific. Furthermore, we did not measure the expression of each protein by Western blotting, and we calculated enzyme activity using total protein amounts. If the proportions of antioxidant enzyme isoform expression were altered in the vitamin E-deficient mice, the respective enzymatic activities could also be greatly altered. In particular, it is known that GPx activity is much lower than that of other antioxidant enzymes in brain. It may be difficult to correctly compare the influence of vitamin E-deficiency when attempting to direct individual differences in mice. The results of antioxidant enzyme activity analysis in the present study were similar to our previous analysis of MAP LC3 protein expression. The antioxidant enzyme activities of the short-term vitamin E-deficient mice did not significantly differ from those in the age-matched controls, since the short-term vitamin E-deficient mice may have been resistant to oxidative stress. However, almost all antioxidant enzyme activities of the long-term vitamin E-deficient mice tended to decrease compared to those in age-matched controls. These results indicate that oxidative damage gradually accumulated in the brains of 6-mo-old vitamin E-deficient mice. Use of a longer-term vitamin $\mathrm{E}$ deficient model, such as 12 mo or more, is more likely to reveal significant differences compared to the age-matched controls. These results, including previous results on autophagy and vitamin E content, were the first studies to clarify the influences of long-term vitamin E-deficiency; however, further investigation is warranted.

The possible role of vitamin $E$ deficiency-induced elevated serum cholesterol in cognitive function

We measured total serum cholesterol to characterize the mechanism of cognitive dysfunction in the long-term vitamin E-deficient mice. Interestingly, total cholesterol levels of vitamin E-deficient mice were significantly increased compared to those of age-matched controls. Comparing the content of normal and vitamin E-deficient diets indicates that the percentage of protein and fat are largely equivalent (protein: normal diet $=18.8 \%$, vitamin E-deficient diet $=16.6 \%$; fat: normal diet $=3.9 \%$, vitamin E-deficient diet $=4.3 \%$; vitamin $\mathrm{E}$ : normal diet $=45.2 \mathrm{mg} / 100 \mathrm{~g}$, vitamin E-deficient diet $=0$; these values are from the information provided by the suppliers, except the vitamin E content of vitamin E-deficient diet, which was detected by HPLC analysis in our laboratory.)

There are many papers focusing on cholesterol content and cognitive function. Kuo et al. reported that treating mice with a high-cholesterol diet enhanced serum and brain cholesterol concentrations, and elevated TBARS content in serum. Furthermore, highcholesterol diet-treated mice exhibited cognitive dysfunction in the Morris water maze test (27). Ettcheto et al. described the relationship between hypercholesterolemia and neurodegeneration using low density lipoprotein (LDL) receptor knockout and amyloid precursor protein (APP)/presenilin (PS) 1 transgenic mice (28). Treatment with $\alpha$-tocopherol attenuated high-cholesterol diet-induced elevation of serum cholesterol level in rabbit models (29). In addition, it is well known that treatment with vitamin $\mathrm{E}$ attenuates the risk of arteriosclerosis by preventing LDL oxidation (30). The elevation of serum cholesterol might be related to an acceleration of cognitive dysfunction via overproduction of oxidative stress in blood. However, we did not analyze the blood components in detail. Furthermore, we have not identified a direct correlation between elevated serum cholesterol and brain oxidation. We are continuing to study the anti-obesity effect of vitamin E using high-fat model mice. In the near future, we aim to determine the relationship between changes in ROS-induced blood components and cognitive dysfunction.

\section{CONCLUSIONS}

In this study, we examined the influence of long-term vitamin E-deficiency in mice. Long-term vitamin E-deficient mice exhibited accelerated cognitive dysfunction compared to the short-term model. Furthermore, the long-term vitamin E-deficiency induced significant lipid peroxidation in the cerebral cortex, cerebellum and hippocampus. Thus, even in a normal environment, vitamin E levels may need to be monitored in order to maintain cognitive function during aging.

\section{Conflict of interest}

The authors confirm that there are no conflicts of interest.

\section{Acknowledgments}

The authors would like to thank Mr. Yuichiro Takahashi for his technical assistance with this research. This work was supported by the Ministry of Education, Culture, Sports, Science, and Technology (MEXT)-Supported Program for the Strategic Research Foundation at Private Universities. This study was also supported by a grant-in-aid for Project Research from the Shibaura Institute of Technology (Tokyo, Japan).

\section{REFERENCES}

1) Stadtman ER, Berlett BS. 1998. Reactive oxygen-mediated protein oxidation in aging and disease. Drug Metab Rev 30: 225-243.

2) Butterfield DA. 2014. The 2013 SFRBM discovery award: selected discoveries from the butterfield laboratory of oxidative stress and its sequel in brain in $\operatorname{cog}$ nitive disorders exemplified by Alzheimer's disease and chemotherapy induced cognitive impairment. Free Radic Biol Med 74: 157-174.

3) Chaturvedi RK, Flint Beal M. 2013. Mitochondrial dis- 
eases of the brain. Free Radic Biol Med 63: 1-29.

4) Sumida S, Niki E, Naito Y, Yoshikawa T. 2013. Involvement of free radicals and oxidative stress in NAFLD/ NASH. Special issue on "Oxidative stress and redox signaling in the gastrointestinal tract and related organs" for Free Radical Research. Free Radic Res 47: 869-880.

5) Begriche K, Massart J, Robin MA, Bonnet F, Fromenty B. 2013. Mitochondrial adaptations and dysfunctions in nonalcoholic fatty acid liver disease. Hepatology $\mathbf{5 8}$ : 1497-1507.

6) Mali VR, Palaniyandi SS. 2014. Regulation and therapeutic strategies of 4-hydroxy-2-nonenal metabolism in heart disease. Free Radic Res 48: 251-263.

7) Elnakish MT, Hassanain HH, Janssen PM, Angelos MG, Khan M. 2013. Emerging role of oxidative stress in metabolic syndrome and cardiovascular diseases: important role of Rac/NADPH oxidase. J Pathol 231: 290-300.

8) Ureshino RP, Rocha KK, Lopes GS, Bincoletto C, Smaili SS. 2014. Calcium signaling alterations, oxidative stress, and autophagy in aging. Antioxid Redox Signal 21: 123-137.

9) Liochev SI. 2013. Reactive oxygen species and the free radical theory of aging. Free Radic Biol Med 60: 1-4.

10) Harman D. 1956. Aging; a theory based on free radical and radiation chemistry. J Gerontol 11: 298-300.

11) Onodera K, Omoi NO, Fukui K, Hayasaka T, Shinkai T, Suzuki S, Abe K, Urano S. 2003. Oxidative damage of rat cerebral cortex and hippocampus, and changes in antioxidative defense systems. Free Radic Res 37: 367-372.

12) Fukui K, Ushiki K, Takatsu H, Koike T, Urano S. 2012. Tocotrienols prevent hydrogen peroxide-induced axon and dendrite degeneration in cerebellar granule cells. Free Radic Res 46: 184-193.

13) Zorov DB, Juhaszova M, Sollott SJ. 2014. Mitochondrial reactive oxygen species (ROS) and ROS induced ROS release. Physiol Rev 94: 909-950.

14) Jiang Q. 2014. Natural forms of vitamin E: metabolism, antioxidant, and anti-inflammatory activities and their role in disease prevention and therapy. Free Radic Biol Med 72: 76-90.

15) Niki E. 2014. Role of vitamin E as a lipid-soluble peroxyl radical scavenger: in vitro and in vivo evidence. Free Radic Biol Med 66: 3-12.

16) Brigelius-Flohe R, Traber MG. 1999. Vitamin E: function and metabolism. FASEB J 13: 1145-1155.

17) Kay MM, Bosman GJ, Shapiro SS, Bendich A, Bassel PS. 1986. Oxidation as a possible mechanism of cellular aging: vitamin $\mathrm{E}$ deficiency causes premature aging and IgG binding to erythrocytes. Proc Natl Acad Sci USA 83: 2463-2467.

18) Niki E, Traber MG. 2012. A history of vitamin E. Ann Nutr Metab 61: 207-212.

19) Fukui K, Onodera K, Shinkai T, Suzuki S, Urano S. 2001. Impairment of learning and memory in rats caused by oxidative stress and aging and changes in antioxidative defense systems. Ann NY Acad Sci 928: 168-175.

20) Fukui K, Omoi NO, Hayasaka T, Shinkai T, Suzuki S, Abe K, Urano S. 2002. Cognitive impairment of rats caused by oxidative stress and aging, and its prevention by vitamin E. Ann NY Acad Sci 959: 275-284.

21) Fukui K, Masuda A, Hosono A, Suwabe R, Yamashita K, Shinkai T, Urano S. 2014. Changes in microtubulerelated proteins and autophagy in long-term vitamin E-deficient mice. Free Radic Res 48: 649-658.

22) Morris R. 1984. Developments of a water-maze procedure for studying spatial learning in the rat. J Neurosci Methods 11: 47-60.

23) Ohkawa H, Ohishi N, Yagi K. 1979. Assay for lipid peroxides in animal tissue by thiobarbituric acid reaction. Anal Biochem 95: 351-358.

24) Chalasani N, Younossi Z, Lavine JE, Diehi AM, Brunt EM, Cusi K, Charlton M, Sanyal AJ. 2012. The diagnosis and management of non-alcoholic fatty liver disease: Practice guideline by the American Association for the study of liver diseases, American College of Gastroenterology, and the American Gastroenterological Association. Hepatology 55: 2005-2023.

25) Yokota T, Igarashi K, Uchihara T, Jishage K, Tomita H, Inaba A, Li Y, Arita M, Suzuki H, Mizusawa H, Arai H. 2001. Delayed-onset ataxia in mice lacking alphatocopherol transfer protein: model for neuronal degeneration caused by chronic oxidative stress. Proc Natl Acad Sci USA 98: 15185-15190.

26) Terada Y, Okura Y, Kikusui T, Takenaka A. 2011. Dietary vitamin $\mathrm{E}$ deficiency increases anxity-like behavior in juvenile and adult rats. Biosci Biotechnol Biochem 75: 1894-1899.

27) Kuo PH, Lin CI, Chen YH, Chiu WC, Lin SH. 2015. A high-cholesterol diet enriched with polyphenols from Oriental plums (Prunus salicina) improves cognitive function and lowers brain cholesterol levels and neurodegenerative-related protein expression in mice. $\mathrm{Br} \mathrm{J}$ Nutr 113: 1550-1557.

28) Ettcheto M, Petrov D, Pedrós I, de Lamos L, Pallás M, Alegret M, Laguna JC, Folch J, Camins A. 2015. Hypercholesterolemia and neurodegeneration. Comparison of hippocampal phenotypes in LDLr knockout and APPswe/PS1dE9 mice. Exp Gerontol 65: 69-78.

29) Bozaykut P, Karademir B, Yazgan B, Sozan E, Siow RC, Mann GE, Ozer NK. 2014. Effects of vitamin E on peroxisome proliferator-activated receptor $\gamma$ and nuclear factor-erythroid 2-related factor 2 in hypercholesterolemia-induced atherosclerosis. Free Radic Biol Med 70: 174-181.

30) Ganini D, Mason RP. 2014. Absence of an effect of vitamin $\mathrm{E}$ on protein and lipid radical formation during lipoperoxidation of LDL by lipoxygenase. Free Radic Biol Med 76: 61-68. 